\title{
Early Diagnosis of Canine Mammary Tumours Using Nipple Aspirate Fluid Cytology and Fine Needle Aspiration Cytological Techniques
}

\author{
P. Nithya, S. Vairamuthu, C. Balachandran, R. Suresh Kumar and \\ B. Murali Manohar
}

\begin{abstract}
In the present study clinical evaluation of canine mammary tumour cases to the Small Animal Surgical Out Patient Ward of the Madras Veterinary College Teaching Hospital, Department of Clinics, Chennai - 600007 between September 2005 and June 2006 was done. During the study period of 10 months, out of 100 dogs screened for mammary gland involvement, 88 showed mammary neoplasms and 12 were nonneoplastic. . NAF (Nipple aspirate fluid) was attempted in 651 glands of 83 dogs, of which, 346 glands of 72 dogs yielded NAF. Out of 346 glands, 224 were asymptomatic NAF yielders were mostly $6-10$ years 44.44 per cent). Pure breeds (73.61 per cent) yielded NAF mostly and 94.44 per cent were intact. There was 30.63 per cent yield from the inguinal glands. NAF was mostly creamy white, dirty, watery and sticky. Cellularity was moderate (52.89 per cent). Immunostained NAF smears revealed high epithelial:macrophage ratio in malignant type. Fine needle aspiration biopsy (FNAB) was taken from 144 glands of 101 dogs including one necropsy case, 93 were malignant and 14 were benign tumours. NAF findings correlated well with FNAB and histopathology in the diagnosis of canine mammary tumours (88.89 per cent).
\end{abstract}

Keywords: Dog , Mammary tumour , NAF , FNAB

\section{Introduction}

Nipple Aspirate Fluid(NAF) cytology is a new, simple, sensitive, quick and non- invasive technique and FNAB is relatively non invasive technique for early detection of canine mammary cancer using cytological and tumour biomarker studies even in non- lactating asymptomatic and suspected dog. The first report of diagnostic use of breast fluid cells was made in 1914(Nathan, 1914). Those samples containing few or no breast epithelial cells are also informative, for they have shown that NAF cytology of low cellularity is associated with low breast cancer risk (Sauter et al., 1997). The utility of NAF cytology with other routine tests in diagnosis of canine mammary tumour was compared by Priya (2005).

\section{Materials And Methods}

Samples were collected from mammary gland of affected dogs presented to Teaching Veterinary Hospital Campus, Madras Veterinary College, Chennai - 600007 during the period of October 2005 to July 2006 by using nipple aspiration and fine needle aspiration biopsy (FNAB). The collected tissue samples were fixed in 95 per cent methanol and stained with Leishman Giemsa stain (SOP - CCL, 2005).Cytological examination was done based on cytologic criteria for NAF was done as described by Priya (2005) and cytologic criteria for FNAB as described by Allen et al .,(1986) and Menard et al., (1986).

\section{Results And Discussion}

In the present study, out of 101 dogs with the mammary gland involvement, NAF was attempted in 651 glands of 83 dogs $(83.17 \%)$, of which $346(53.15 \%)$ of 72 dogs yielded NAF . Among 346 glands, 122 $(35.26 \%)$ were tumour bearing glands and 224(64.74\%) were asymptomatic. Age wise incidence: The $6-10$ years age group yielded NAF mostly with a mean age of 8.5 years Breed wise incidence : Pure breeds $(73.61 \%)$ yielded NAF mostly followed by non-descript $(25.00 \%)$ and cross-bred $(1.39 \%)$ dogs. Among the pure breeds, the highest NAF yielder was Spitz $(n=14)$, followed by Doberman $(n=10)$ and German shepherd $(n=8)$. Reproductive status: Among the NAF yielders, 4 were spayed (5.56\%) and the remaining dogs were intact (94.44\%). Of these animals, 33 had whelped and 39 were not-bred. Gland wise Incidence: Inguinal glands yielded mostly $(\mathrm{n}=106)$ followed by caudal abdominal glands $(\mathrm{n}=102)$. Out of 346 glands which yielded NAF, $224(64.74 \%)$ were asymptomatic and $122(35.26 \%)$ had palpable masses.

Categories of NAF samples were presented in Table (1) 
Table 1 Categories of NAF Samples

\begin{tabular}{|l|l|l|}
\hline Tumour types & No. of samples & Per cent \\
\hline Benign & 38 & 10.98 \\
\hline Malignant & 203 & 58.67 \\
\hline Atypical & 46 & 13.29 \\
\hline Inflammatory & 47 & 13.58 \\
\hline Non-diagnostic / no cellularity & 12 & 3.47 \\
\hline Total & $\mathbf{3 4 6}$ & $\mathbf{9 9 . 9 9}$ \\
\hline
\end{tabular}

Out of 346 gland samples 38 (10.98\%) were benign, 203 (58.67\%) malignant, 46 (13.29\%) atypical, $47(13.58 \%)$ inflammatory and $12(3.47 \%)$ were non- diagnostic/ no cellularity. Cytologic criteria of benign tumours were loose or clusters in well organised acinar pattern, cuboidal cells with round and smooth contoured nuclei, fine chromatin, cytoplasmic vacuolation showed signet ring type of cells with flattened eccentric nuclei. Atypical category showed less organised cell clusters, papillary like clusters and cells showed anisocytosis, eccentric nucleus with anisokaryosis and coarse chromatin, basophilic cytoplasm, nucleoli were large, nuclear to cytoplasm ratio increased. Malignant category revealed large clusters with less organised, contained hyperchromatic nuclei with coarse chromatin, mitotic figures with multinucleated giant cells were also observed. Emperipolesis was also found.

In the present study, NAF was obtained in 53.15 per cent glands of 72 dogs $(71.29 \%)$ which is higher than that reported by Priya (2005). NAF yield from the 6-10 years age group of pure breed dogs was the highest (73.61\%). NAF was obtained mostly from intact animals $(94.44 \%)$ with highest yield from inguinal glands (30.64\%) followed by caudal abdominal glands $(29.48 \%)$. NAF was mostly creamy white $(42.20 \%)$ or dirty $(28.32 \%)$ in colour. Of the 346 glands which yielded NAF, $52.89 \%$ had moderate cellularity. These findings are in accordance with the findings of Priya (2005). The foam cells were higher in number, 54.73, 46.90 and 51.00 per cent in benign, atypical and malignant than the non-foam cells $20.80,16.00$ and 9.20 per cent respectively. The high percentage of PMN in malignant and atypical type $(32.00 \%$ and $29.00 \%)$ indicated the presence of concurrent infection and necrosis. The non-foam cell: foam cell ratio was higher in benign (0.38), followed by atypical (0.33) and malignant categories (0.18). Exfoliation was recorded in our study which is in accordance with several works which might be due to involutional phenomenon. The ratio of epithelial cells to foam cells ranged from 0.09 to 3.4 with a median value of 0.8 is in agreement with earlier report of (King et al., 2002). Clusters were recorded more in malignant tumours which are in accordance with Priya, 2005. Epithelial origin of malignant cells detach frequently in clusters (Fleury et al., 1994).

\section{FNAB}

FNAB was taken from 101 dogs involving 144 glands, of which 22 were inflammatory, 14 benign, atypia, 93 malignant and 14 non- diagnostic(Table 2)

Table 2 FNAB findings in mammary tumour dog

\begin{tabular}{|l|c|c|}
\hline \multicolumn{1}{|c|}{ Conditions } & No. of cases & Per cent \\
\hline Mastitis & 22 & 15.28 \\
\hline Benign & 14 & 9.72 \\
\hline Malignant & 93 & 64.59 \\
\hline Cyst & 1 & 0.69 \\
\hline Non-diagnostic & 14 & 9.72 \\
\hline \multicolumn{1}{c|}{ Total } & $\mathbf{1 4 4}$ & $\mathbf{1 0 0}$ \\
\hline
\end{tabular}

Tubular adenocarcinoma simplex showed high cellularity, multiple large clusters, multipapillary clusters, Anisokaryosis, round caudate cells, spherical to oval nuclei, hyperchromatism, binucleate to multinucleate cells with coarse chromatin, N:C ratio high with emperipolesis. In the complex tumours, epithelial clusters were surrounded by spindle shaped cells containing oval to elongated nuclei with indistinct cell borders. Simplex papillary adenocarcinoma had papillary pattern, solid sheet of cells in solid carcinoma, Spindle, fusiform cells in spindle cell carcinoma, polyhedral squamoid cells with prominent nucleoli in squamous cell carcinoma.

In carcinosarcomas, epithelial clusters with spindle shaped cells were observed. In benign tumours, epithelial tumours contained small to large clusters containing more or less uniform cells with round and uniform nucleus. Lipoma showed polyhedral vacuolated cells with eccentric nuclei. Adenocarcinomas observed in the present study exhibited malignant cytological criteria which is in accordance with Coles (1986). From this study, NAF findings correlated well with FNAB and histopathology in the diagnosis of canine mammary tumours (88.89 per cent) 


\section{Summary}

In the present study, the highest yield of NAF was obtained in $6-10$ years (44.44\%) age group with highest in pure breeds (73.61\%) followed by non- descript (25.00\%) dogs. Among the NAF yielders, $94.44 \%$ were intact. Posterior glands $(60.11 \%)$ yielded NAF more than other glands. NAF was mostly creamy white $(42.2 \%)$ and dirty $(28.32 \%)$ in colour. Cellularity was moderate $(52.89 \%)$. NAF were categorized into benign $(\mathrm{n}=38)$, atypical $(\mathrm{n}=46)$ and malignant $(\mathrm{n}=203)$ based on cytological features in malignancy. Differential cell count scoring on non - immunostained smears showed more foams than non - foam cells. Number of cells in each cluster was high in malignant type. FNAB was obtained from 144 glands, of which 64.59 per cent were malignant. NAF findings had good correlation (88.89\%) with FNAB and histopathology and therefore useful in the early diagnosis of canine mammary tumours.

\section{References}

[1]. Allen. S.W., K.W. Prasse and E.A. Mahaffey. 1986. Cytologic differentiation of benign from malignant canine mammary tumours. Vet. Pathol., 23: 649-655

[2]. Coles, E.H. 1986. Veterinary Clinical Pathology, 4th Edn. W.B. Saunders Company, Philadelphia.

[3]. Fleury, C.F., J.P. Magnol and J.F. Guelfi, 1994. In color atlas of cancer cytology of the dog and cat. Conference Nationale des Veterinaries Specialises en Petitis Animaux, Paris. pp.29.

[4]. King, B.L., G.M. Crisi, S.V. Tsai, B.G. Haffy, R.F. Philips and D.L. Rimm. 2002. Immunocytochemical analysis of breast cells obtained by ductal lavage. 96 (4): 244-249.

[5]. Menard, M., M. Fontaine and M. Morin. 1986. Fine needle aspiration biopsy of malignant tumours in dogs and cats. Can. Vet. J., 27: 504-510.

[6]. Nathan, M. 1914. Diagnosgtic prococe d'un neoplasme du sein par l'examen histologique di son suintement hemorragique clinique paris. 60: 38 - 69 .

[7]. Priya, S. 2005. Comparison of utility of nipple aspirates fluid cytology with other routine diagnostic tests. M.V.Sc., thesis submitted to TANUVAS, Chennai - 600051.

[8]. Sauter, E.R., E. Ross, M.Daly, A. Klein-zanto, P.F. Engstrom, A. Sorling, J. Malick and H. Ehya. 1997. Nipple aspirate fluid : a promising non-invasive method to identify cellular markers of breast cancer risk. Br. J. Cancer., 76 (4) : $494-501$.

[9]. SOP-CCL. 2005. Standard Operating Procedures. Centralised Clinical Laboratory, Madras Veterinary College, Chennai - 600007. 